\title{
A Case of Mistaken Inflammatory Pseudotumor
}

\author{
${ }^{1}$ Steven K Dennis, ${ }^{2}$ Kevin J Caceres, ${ }^{3}$ William C Yao
}

\begin{abstract}
Aim: Inflammatory myofibroblastic pseudotumor (IMT), part of the inflammatory pseudotumor (IPT) family, shares many presenting features with more common expansile sinonasal masses and is unlikely to be diagnosed without clinical suspicion.
\end{abstract}

Background: Inflammatory myofibroblastic pseudotumor (IMT) is histologically characterized by myofibroblasts with prominent small lymphocytes and plasma cells, often initially mistaken for other inflammatory processes. These lesions share characteristics of pseudotumors and neoplasms with possible malignant potential.

Case description: We present the case of a 57-year-old woman who was eventually properly diagnosed with IMT. She initially presented with several month histories of severe right-sided headache, facial pain, blurry vision, and right lid ptosis. The patient was diagnosed with invasive fungal sinusitis by another provider but proved nonresponsive to intravenous antifungal therapy. Repeat biopsy revealed an inflammatory process consistent with IMT. The lesion was rapidly responsive to intravenous steroid therapy.

Conclusion: A destructive sinonasal mass necessitates a wide differential diagnosis that should include IPT. Both medical and multimodal treatment strategies have proven highly efficacious with a high cure rate and a higher rate of tumor stabilization.

Clinical significance: IMT is an example of a commonly misdiagnosed pathology even with permanent section analysis by several pathologists. Early identification of this condition can lead to better treatment with medical therapeutic options and minimize unnecessary interventions.

Keywords: Biologic therapy, Corticosteroids, Invasive fungal sinusitis, Inflammatory myofibroblastic pseudotumor, Inflammatory pseudotumor.

How to cite this article: Dennis SK, Caceres KJ, Yao WC. A Case of Mistaken Inflammatory Pseudotumor. Clin Rhinol An Int J 2018;11(1):21-24.

Source of support: Nil

Conflict of interest: None

${ }^{1,2}$ Resident, ${ }^{3}$ Assistant Professor

${ }^{1}$ Department of Otolaryngology, University of California Davis, Sacramento, California, USA

${ }^{2,3}$ Department of Otorhinolaryngology, University of Texas McGovern Medical School at Houston, Houston, Texas, USA

Corresponding Author: William C Yao, Assistant Professor, Department of Otorhinolaryngology, University of Texas McGovern Medical School at Houston, Houston, Texas, USA, e-mail: william.c.yao@uth.tmc.edu

\section{BACKGROUND}

Inflammatory pseudotumor (IPT) was first described by Busse and Hockheim in 1903 and received its first histopathological description by Birch-Hirschfield in $1905 .{ }^{1}$ IPT encompasses non-neoplastic inflammatory processes characterized by fibroblastic and myofibroblastic proliferation in a background of chronic inflammatory infiltrate with several subtypes. ${ }^{1,2}$ IPT shares presenting features with multiple more common diagnoses, including nonspecific constitutional symptoms with possible mass effect from the growing tumor, difficult to distinguish from malignancy. ${ }^{1,3}$ Primarily characterized as a nonspecific lesion with chronic inflammatory changes, a controversial subset has emerged, IMT. ${ }^{4}$ These lesions share characteristics of pseudotumors and neoplasms with possible malignant potential.

In the head and neck, IPT is less likely to exhibit systemic symptoms and shows variable inflammatory markers. ${ }^{3}$ Sinonasal and ventral skull base IPT most commonly present with vision changes and diplopia, followed by a headache and facial pain, or epistaxis and nasal obstruction. ${ }^{5}$ Cranial nerve palsy is another common presenting symptom, with CN VI, CN V and CN III being the most frequently affected, in relation to the propensity for the cavernous sinus. ${ }^{1} \mathrm{IMT}$ has rare distant metastatic potential and has a higher likelihood of being aggressive and locally invasive than other types of IPT. The World Health histologic definition of IMT is a space-occupying lesion of myofibroblasts accompanied by prominent small lymphocytes and plasma cells. ${ }^{6}$ The most common extraorbital site of head and neck presentation is the maxillary sinus. ${ }^{1,5}$

In this report, we present a case of sinonasal and ventral skull base IMT that presented with a right-sided headache and right atypical facial pain. Before collecting patient information, an IRB exemption (HSC-MS-15-0642) was obtained.

\section{CASE DESCRIPTION}

A 57-year-old woman with a past medical history of smoking, hypertension, hypothyroidism, and major depressive disorder originally presented to a different surgical team with a $4-5$ months history of severe rightsided headache with right facial pain and was diagnosed with acute sinusitis. While being treated with IV antibiotics, she developed acute worsening of blurry vision and 
right lid ptosis over a 2-week period. She denied systemic symptoms, nasal congestion, nasal drainage, or changes in smell. Flexible fiberoptic nasal endoscopy revealed a right sinonasal mass. A CT maxillofacial scan revealed right-sided pansinus opacification and significant bony erosion of the right medial orbital wall and fovea ethmoidalis with possible intracranial extension. An MRI exhibited a sinonasal mass in the right ethmoid sinus that extended into the orbit with lateral displacement of the medial and inferior recti muscles as well as an intracranial extension (Fig. 1A). There was no evidence of meningeal enhancement or extension into the cavernous sinus.

The patient was taken to the operating room (OR) for endoscopic sinus surgery and endoscopic biopsy of the mass. The patient was noted to have inflamed, edematous and friable tissue within the right posterior ethmoid sinuses involving the right medial orbital wall, optic nerve, and orbital apex. The original surgeon noted a lack of any discernible masses and a presumed diagnosis of invasive fungal sinusitis was made. The pathological diagnosis noted the presence of severe chronic sinusitis with some areas of necrosis and granulomatous inflammation as well as some fungal elements, which was thought to confirm the presumed diagnosis. The patient was treated with antifungals and antibiotics as recommended by the infectious disease team.

Two months after initial presentation, the patient presented to our service with a recurrence of symptoms. Repeat imaging revealed the progression of the right sino-
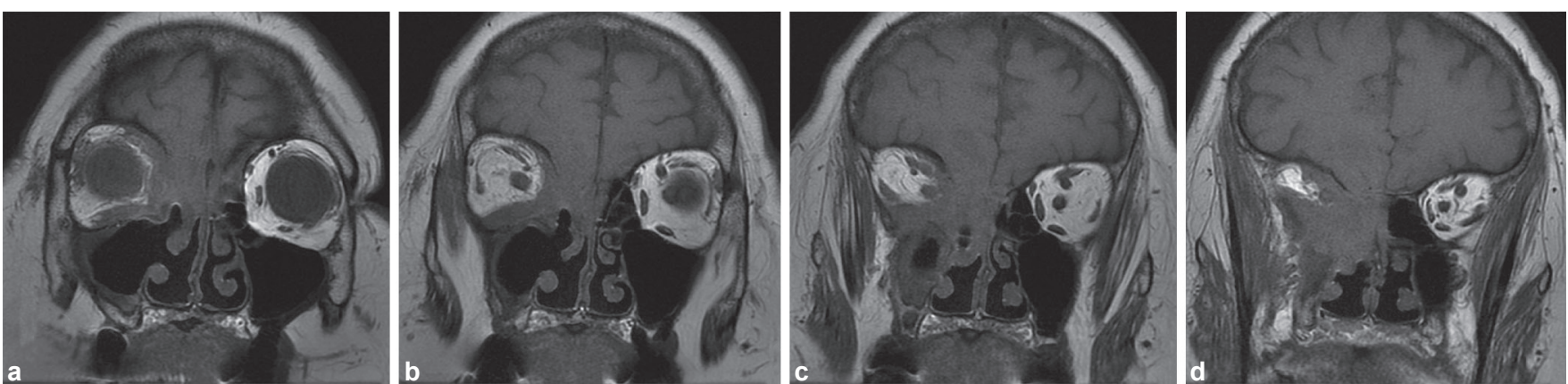

Fig. 1A: T1-weighted coronal MRI revealing the tumor on the right orbit along the orbit and skullbase which were obtained at the time of original diagnosis.(a to d) represent coronal sections from anterior to posterior
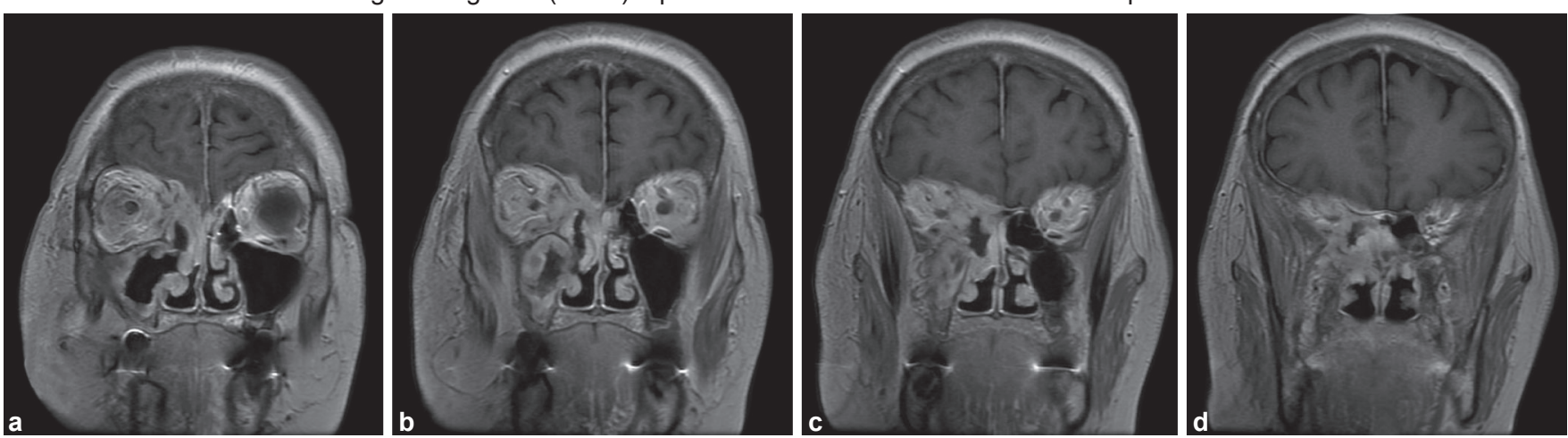

Fig. 1B: T1-weighted coronal MRI revealing the tumor on the right orbit along the orbit and skull-base which were obtained 4 months following steroid administrations showing improvement from Figure 1A. (a to d) represent coronal sections from anterior to posterior 

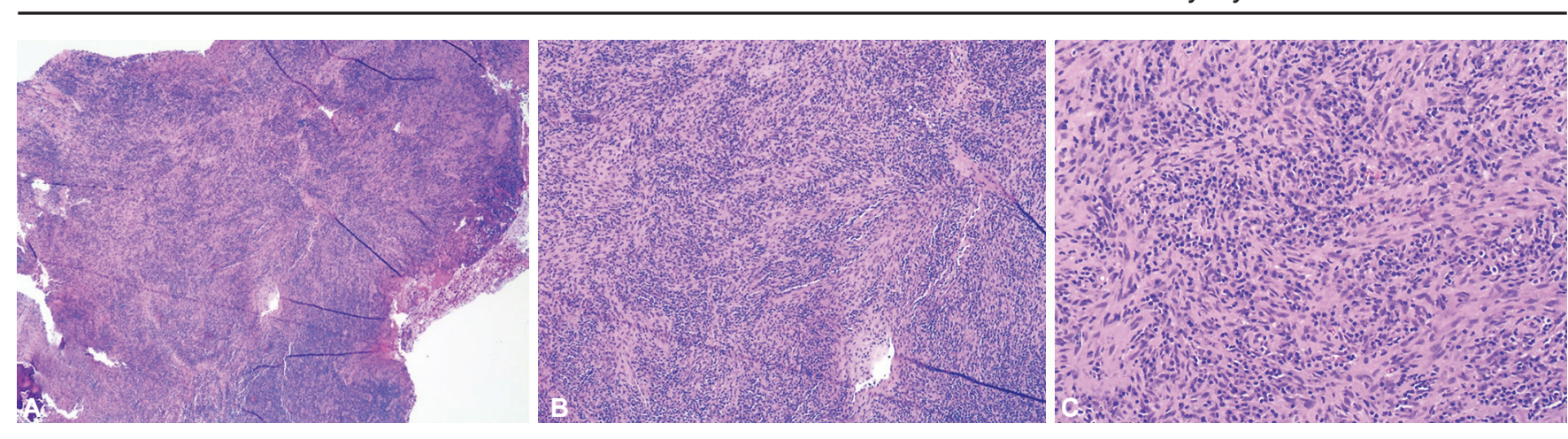

Figs 2A to C: H\&E biopsy from posterior orbital wall showing dense lymphoplasmacytic infiltrates with proliferation of spindle cells. (A) Low-power view; (B) Mid-power view; (C) Higher-power view
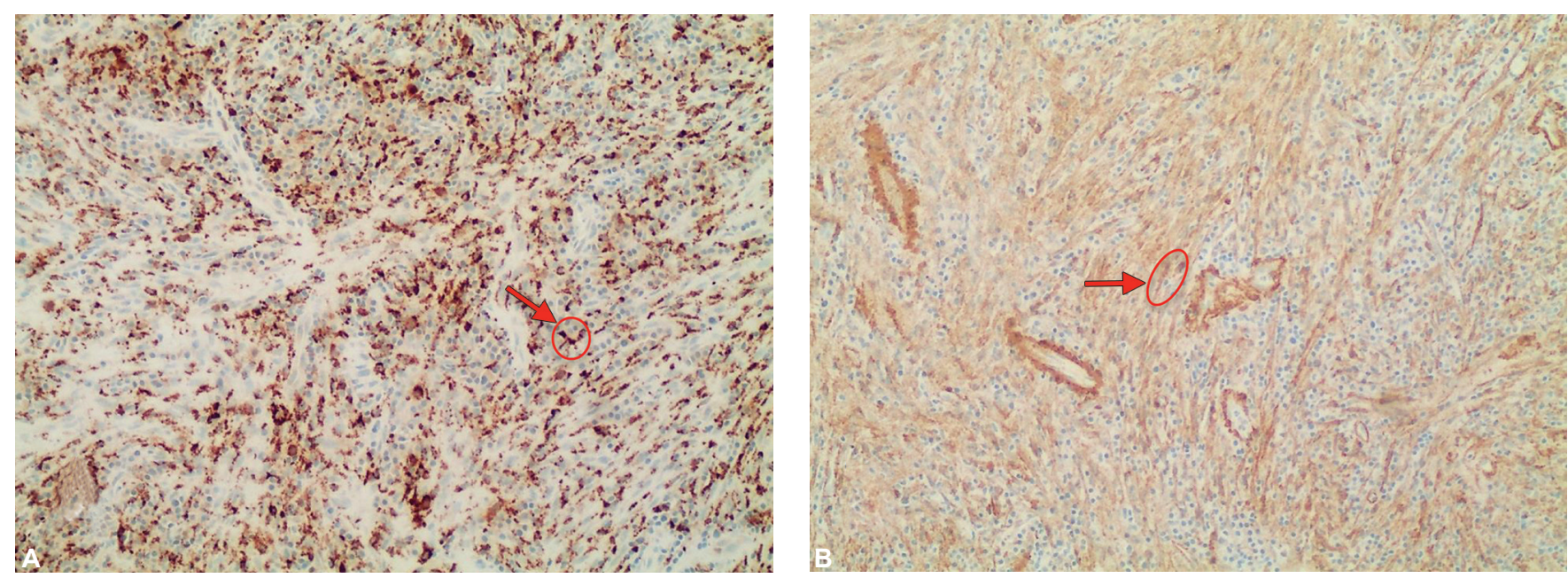

Figs 3A and B: Selected immunohistochemical stains. (A) CD68 positive histiocytes; (B) SMA positive myofibroblasts

images. ${ }^{1}$ Moreover, there will be evidence of local invasion with bony erosion and sclerosis. ${ }^{2}$ Laboratory tests are an effective adjunct to imaging, with ESR and CRP elevation in many cases of IPT, including head and neck. ${ }^{1}$ Leukocytosis, anemia, and hypergammaglobulinemia are also possible lab findings.

Clinical diagnosis requires a biopsy. Awareness of IPT and inclusion in the differential is critical to diagnosis. The histopathology of IPT exhibits fibrosis, spindle cells, and the presence of inflammatory cells without the presence of bacteria, fungi or cellular atypia. The differential for spindle cells and inflammatory infiltrate includes multiple benign and malignant etiologies. ${ }^{6}$ Definitive diagnosis of IMT currently entails the identification of a rearrangement of anaplastic lymphoma kinase (ALK) gene by fluorescence in situ hybridization which occurs in approximately $50 \%$ of all IMTs as opposed to other forms of IPT which are characterized by the presence of IgG4. ${ }^{6,7}$ Even with biopsy, significant experience with the morphologic features of IPT is critical to recognize the disease. ${ }^{5}$ Diagnosis is usually achieved after multiple excisional biopsies and close examination of histological features.

In our patient, there was evidence of fungal elements on the original biopsy by a different service that led to the incorrect treatment pathway. Ponikau et al. collected and cultured the nasal mucus of chronic sinusitis (CRS) patients as well as healthy control patients. Of the healthy controls, $100 \%$ of the 14 patients had a fungal positive culture as well as $96 \%$ of the CRS patients. ${ }^{8}$ Later studies by Catten et al. utilizing polymerase chain reaction (PCR) techniques have similarly shown no statistical difference between the fungal presence in healthy and CRS patients. ${ }^{9}$ Fungus is a strongly correlated yet poorly understood pathophysiologic factor in chronic rhinosinusitis. However, its presence should not lead to errant conclusions. Therefore, it is important to communicate closely with the pathology department because the original specimen can easily be misinterpreted without a clinical suspicion.

The treatment strategy for IPT continues to evolve, but medical therapy has remained the mainstay treatment. In some cases, surgical excision with clear margins may be considered. However, the tumor location and possible surgical morbidity must be taken into account. ${ }^{6}$ Some tumors have exhibited complete resolution with the administration of steroids, but more commonly their progression is only halted by steroid use. ${ }^{1}$ Orbital IPT has been shown to be especially responsive to steroids, while sinonasal IPT has shown to be less responsive. ${ }^{10}$ Early recognition and diagnosis can be vital in avoiding radical ablative surgery because of the 
lack of good medical response. Radiotherapy and/or chemotherapy have been employed in a few cases of highly invasive or inoperable IMT with mixed results, often showing resistance.

Imaging is currently being investigated for its predictive ability for the efficacy of steroid therapy. ${ }^{2}$ IPT with strong T2 signaling with contrast-enhanced imaging indicates an acute inflammatory process that will be more responsive to steroids. Poor T2 signaling may indicate fibrosis and poor steroid response, which would make better surgical candidates. Amongst all treatment modality outcomes, Desai et al. noted that $62.1 \%$ of patients had perpetuance of active disease, while only $27.6 \%$ had no evidence of disease following treatment. ${ }^{1}$ Other studies have shown the local recurrence rate to be approximately $10-20 \%$ after surgical resection alone. ${ }^{6}$

There has been limited investigation into targeted therapies. Crizotinib, a molecular-targeted therapy against ALK was investigated in two patients with intra-abdominal IMT. ${ }^{7}$ The study did show the promising result with no evidence of recurrence following surgical resection and targeted therapy. However, these types of studies have yet to be investigated for head and neck cases.

\section{CONCLUSION}

As seen in our case, it is important to keep a wide differential diagnosis and consider IPT for a destructive sinonasal mass. IPT is unlikely to be diagnosed histologically without clinical suspicion and moreover, the concern should be shared with the pathology department. Early recognition and diagnosis can significantly decrease the treatment course for these patients. Depending on the accessibility of the lesion, the feasibility and efficacy of surgical excision should be assessed for each patient. While the highest cure rate is seen with combined treatment strategies, steroid therapy alone has shown positive effects with a high cure rate and a higher rate of tumor stabilization.

\section{CLINICAL SIGNIFICANCE}

Inflammatory myofibroblastic tumor (IMT) is an example of a commonly misdiagnosed pathology even with permanent section analysis by several pathologists. Early identification of this condition can lead to better treatment with medical therapeutic options and minimize unnecessary interventions.

\section{ACKNOWLEDGMENT}

Institutional IRB exemption (HSC-MS-15-0642) was obtained.

\section{REFERENCES}

1. Desai SV, Spinazzi EF, Fang $\mathrm{CH}$, et al. Sinnasal and Ventral Skull Base Inflammator Pseudotumor: A Systematic Review. The Laryngoscope 2015;125:813-821.

2. Lai LM, McCarville MB, Kirby $\mathrm{P}$, et al. Shedding light on inflammatory pseudotumor in children: spotlight of inflammatory myofibroblastic tumor. Pediatric Radiology, Published online:12 May 2015.

3. Soysal V, Yigitbasi OG, Kontas O, et al. Inflammatory myofibroblastic tumor of the nasal cavity: a case report and review of the literature. Int J Pediatr Otorhinolaryngol, 2001;61:161165.

4. Tao J, Zhou ML, Zhou SH. Inflammatory myofibroblastic tumors of the head and neck. Int J Clin Exp Med 2015;8(2):1604-1610.

5. Huang WH, Dai YC. Inflammatory Pseudotumor of the nasal cavity. Am J Otolaryngol 2006;27:275-277.

6. Devaney KO, LaFeir DJ, Triantafyllou A, et al., Inflammatory myofibroblastic tumors of the head and neck: evaluation of clinicopathologic and prognostic features. Eur Arch Otorhinolaryngol 2012;269(12):2461-2465.

7. Butrynski JE, D'Adamo DR, Hornick JL, et al., Crizotinib in ALK-Rearranged Inflammatory Myofibroblastic Tumor. New Eng J Med 2010;363(18):1727-1733.

8. Ponikau JU, Sherris DA, Kern EB, et al., The diagnosis and incidence of allergic fungal sinusitis. Mayo Clin Proc 1999;74(9):877-884.

9. Catten MD, Murr AH, Goldstein JA, et al., Detection of fungi in the nasal mucosa using polymerase chain reaction. Laryngoscope 2001;111(3):399-403.

10. Park SB, Lee JH, Weon YC. Imaging Findings of Head and NEck Inflammatory Pseudotumor. Am J Roentgenol 2009;193:1180-1186. 